\title{
Perfil clínico e nutricional de crianças com alergia à proteína do leite de vaca
}

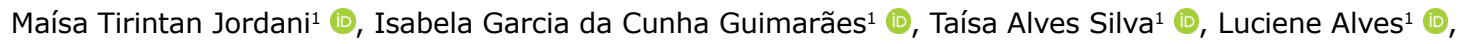
Camila Bitu Moreno Braga ${ }^{1}$ (D), Sylvana de Araújo Barros Luz ${ }^{1}$ (D)

\section{RESUMO}

Objetivo: Este estudo teve como objetivo identificar as principais características clínicas e nutricionais apresentadas pelas crianças com alergia à proteína do leite de vaca. Método: Tratou-se de um estudo observacional, transversal, quantitativo e correlacional, com amostra de conveniência, constituída por 22 crianças diagnosticados com alergia em diferentes estágios, na faixa etária de 6 meses a 6 anos, atendidas no ambulatório de Gastroenterologia Pediátrica do Hospital de Clínicas da Universidade Federal do Triângulo Mineiro. Resultados: Evidenciou-se eutrofia em 81,8\% das crianças, risco de sobrepeso em 4,5\%, sobrepeso em $9,1 \%$ e obesidade em $4,5 \%$. Nenhum dos pacientes apresentou diagnóstico nutricional de magreza. Os sintomas gastrointestinais (diarreia, vômito e sangue vivo nas fezes) foram os que mais acometeram os pacientes avaliados. Todos os pacientes $(100 \%)$ com alergia em remissão e alergia resolvida mostraram nível de ferritina sérica normal, porém, $14,3 \%$ das crianças em alergia ativa estavam com esse valor abaixo do recomendado para idade. As crianças apresentam desmame precoce do leite materno, alimentação complementar dentro do que é indicado e o uso de fórmulas infantis corretas para atingir as recomendações nutricionais para a idade. Conclusão: Os achados indicam que o perfil clínico e nutricional de crianças com alergia está em conformidade com o esperado no que tange à escolha das fórmulas e desenvolvimento das crianças.

Palavras-chave: Alergia, Leite de vaca, Nutrição, Saúde. 


\section{INTRODUÇÃO}

A alergia alimentar é uma reação adversa que ocorre quando o sistema imunológico reconhece um alimento como uma substância agressora ao organismo, sendo ele então definido como alérgeno. ${ }^{1}$ Os alérgenos alimentares são definidos como componentes de alimentos ou ingredientes dentro de alimentos reconhecidos por células imunes específicas provocando reações imunológicas e sintomas característicos. ${ }^{2}$ Entre os alimentos, o leite de vaca merece ênfase nos casos de alergia alimentar em crianças, já que, habitualmente é o primeiro alimento a ser ofertado, sendo a alergia à proteína do leite de vaca (APLV) uma das principais causas de alergia alimentar em lactentes e crianças menores de três anos. ${ }^{3}$

Atualmente, sua taxa de prevalência aumentou em cerca de $20 \%$ nos últimos 10 anos. ${ }^{4} \mathrm{Um}$ estudo realizado em serviços públicos de atenção nutricional, de 34 municípios brasileiros, constatou prevalência de $0,4 \%$ de APLV em lactentes e crianças pequenas. 5 Um trabalho elaborado por gastroenterologistas pediátricos apontou uma incidência de APLV de 2,2\% e a prevalência de $5,4 \%$ em crianças atendidas nos serviços do hospital da pesquisa. ${ }^{6}$

Entre os fatores que podem se relacionar à ocorrência da APLV, há fatores intrínsecos, como a hereditariedade, e fatores extrínsecos, como a interrupção precoce do aleitamento materno, revelando ser a amamentação fator essencial na proteção à saúde da criança e prevenção de doenças como a APLV. 7,8

Dentre os alérgenos responsáveis pelas reações decorrentes da APLV, destaca-se a caseína, a alfa-lactoalbumina e a beta-lactoglobulina. ${ }^{9,10,11,12}$ Os fatores relacionados à sensibilização e tolerância à proteína do leite de vaca envolvem predisposição genética, infecções e alteração da microbiota intestinal, idade da primeira exposição, dieta materna, quantidade e frequência da ingestão do alérgeno. ${ }^{3}$ Essa alergia pode ser classificada em três categorias: mediada por imunoglobulina $\mathrm{E}$ (IgE), não mediada por IgE e mista. ${ }^{13,14} \mathrm{Em}$ cada uma delas, os sintomas se manifestam de maneira diferente, variando em alterações cutâneas, problemas gastrointestinais, respiratórios, sistêmicos e até cardiovasculares, sendo os três primeiros os mais comuns. ${ }^{4}$

A maioria dos casos de APLV são diagnosticados ainda na lactação, no momento de substituição do leite materno por fórmulas lácteas ou na introdução da alimentação complementar, sendo esse período da vida em que se observa rápida velocidade de crescimento e desenvolvimento, enfatizando, assim, a elevada relevância da doença. ${ }^{15,16}$ Um dos tratamentos mais eficaz até hoje para pacientes alérgicos à proteína do leite de vaca é a dieta de exclusão, no entanto, essa eliminação deve ser feita com cautela, visto que pode resultar em efeitos colaterais importantes, como desnutrição, deficiência de cálcio, ferro ou outros micronutrientes e macronutrientes, além de alterações no hábito alimentar. ${ }^{9,17,18,19}$ Ao excluir o leite de vaca, é necessário realizar a substituição deste alimento para as crianças menores de seis meses ou então a complementação para as maiores de seis meses, que no caso, é feita por fórmulas à base de proteína isolada de soja, de proteínas extensamente hidrolisadas ou de aminoácidos, a depender de critérios clínicos. ${ }^{20,21,22}$

Baseado nestas considerações, este estudo teve como objetivo identificar as principais características clínicas e nutricionais apresentadas pelas crianças com APLV em diferentes estágios, atendidas no Ambulatório de Pediatria do Hospital de Clínicas da Universidade Federal do Triângulo Mineiro (HC-UFTM).

\section{MATERIAIS E MÉTODOS}

Trata-se de estudo observacional, transversal, quantitativo e correlacional. A amostra de conveniência foi constituída por 22 crianças diagnosticados com APLV, na faixa etária de 6 meses a 6 anos, atendidas no ambulatório de Gastroenterologia Pediátrica do Hospital de Clínicas da Universidade Federal do Triângulo Mineiro (HC-UFTM). Os participantes foram alocados em três grupos de acordo com a condição da APLV, ou seja, alergia ativa $(n=7)$, alergia resolvida $(n=12)$ e alergia em remissão $(n=3)$. Foram excluídos da pesquisa os pacientes com APLV em uso sonda enteral para alimentação.

A coleta de dados foi realizada entre o período de julho de 2018 a dezembro de 2018. Os dados socioeconômicos, com exceção da renda familiar, o valor de ferritina sérica e o uso de suplementação de sulfato ferroso foram coletados a partir do prontuário dos participantes da pesquisa. A data dos resultados dos exames de ferritina sérica das crianças avaliadas foi entre março a outubro de 2018. As informações quanto à renda familiar, ao histórico alimentar 
(presença e duração de aleitamento materno exclusivo, e idade da introdução alimentar) e as características clínicas apresentadas pelos pacientes (sintomas, idade do início dos sintomas, idade de confirmação do diagnóstico, tipo e fase da alergia, tipo de preparação consumido para o aparecimento dos sintomas e substituto do leite de vaca utilizado) foram identificadas por questionário semiestruturado aplicado aos responsáveis legais acompanhantes das crianças. O valor de ferritina sérica de cada paciente foi classificado de acordo com os valores de referência do Laboratório Análises Clínicas do HC-UFTM para a respectiva faixa etária.

Para avaliar o estado nutricional, foram utilizados os padrões de crescimento da Organização Mundial da Saúde (OMS), a partir da análise do escore $Z$ do índice de massa corpórea (IMC) pela idade. Tendo em vista os valores de corte preconizados, as crianças foram classificadas em estado de magreza, eutrofia, sobrepeso ou obesidade. ${ }^{23}$ Para a análise dos dados utilizaram-se os programas WHOAnthro ${ }^{\circledR}$ e WHOAnthroPlus ${ }^{\circledR}$.

Para investigar a ingestão alimentar, utilizouse questionário de frequência alimentar validado para crianças de 2 a 5 anos. ${ }^{24}$ Foram selecionados para a análise neste estudo, os alimentos fonte de ferro heme, ou seja, alimentos de fonte animal e de melhor absorção no organismo, os alimentos industrializados e as hortaliças. O consumo em número de porções de cada um desses grupos alimentares foi comparado às recomendações para cada faixa etária da infância. ${ }^{25}$ Analisou-se, também, a frequência de consumo de alimentos que contém ou podem conter leite de vaca (LV) em sua composição pelos pacientes que apresentavam alergia ativa, em remissão ou resolvida no momento da entrevista.

Foram utilizados os softwares, programa STATISTICS $6.0^{\circledR}$ (StatSoft Incorporation, Tulsa, Ok, USA) e o Statistical Package for the Social Sciences (SPSS) versão 17.0, para todas as análises descritivas e estatísticas, sendo fixado um nível de significância de $5 \%(p<0,05)$.

O estudo foi aprovado pelo Comitê de Ética em Pesquisa da Universidade Federal do Triângulo Mineiro (UFTM) com protocolo n. ${ }^{0}$ 2.669.707, conferido todos os parâmetros éticos exigidos por lei.

\section{RESULTADOS}

Participaram do estudo 22 crianças com idade média de $2,87 \pm 1,71$ anos, sendo $63,6 \%$ do sexo masculino e $36,4 \%$ do feminino; $63,6 \%$ se autodenominaram pelos seus respectivos responsáveis legais de cor branca e 36,4\% de cor parda; $63,6 \%$ eram procedentes da cidade de Uberaba (MG) e $36,4 \%$ de demais cidades da região. Quanto à renda familiar, foi identificado que $36,4 \%$ obtinham a renda de um salário mínimo, $31,8 \%$ de três ou mais salários mínimos, $27,3 \%$ de dois salários mínimos e apenas 4,5\% tinham a renda inferior a um salário mínimo.

A idade média de início dos sintomas de APLV foi

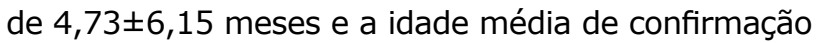

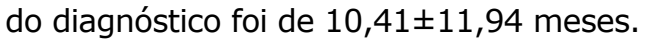

Dentre os pacientes, $50 \%$ apresentaram o tipo de alergia mediada por imunoglobulina $E$ (IgE) e $40,9 \%$ o tipo de alergia não mediada por IgE (tipo celular), e somente $9,1 \%$ possuíam o tipo de alergia mista (celular e mediada por IgE).

Os sintomas apresentados por todas as crianças $(100 \%)$ eram devido ao consumo de LV in natura. Os derivados do LV, o LV adicionado em preparações cozidas ou assadas e os alimentos processados que contém LV na sua composição causavam sintomas em $54,5 \%, 50 \%$ e $45,5 \%$ dos pacientes, respectivamente.

Como observado na Tabela 1 , os sintomas gastrointestinais foram os que mais acometeram os pacientes avaliados. Dentre esses sintomas, os tipos mais citados pelos responsáveis foram diarreia, vômito e sangue vivo nas fezes.

Tabela 1. Comparação das manifestações conjugadas e isoladas apresentadas pelas crianças com APLV, atendidas no Ambulatório de Pediatria do Hospital de Clínicas da Universidade Federal do Triângulo Mineiro, Uberaba (2018). $(n=22)$

\begin{tabular}{|c|c|c|c|}
\hline \multicolumn{2}{|c|}{ Manifestações conjugadas } & \multicolumn{2}{|c|}{ Manifestações isoladas } \\
\hline $\begin{array}{l}\text { Manifestações } \\
\text { gastrointestinais, } \\
\text { respiratórias e } \\
\text { de pele }\end{array}$ & $31,81 \%$ & $\begin{array}{l}\text { Manifestações } \\
\text { gastrointestinais }\end{array}$ & $18,18 \%$ \\
\hline $\begin{array}{l}\text { Manifestações } \\
\text { gastrointestinais } \\
\text { e respiratórias }\end{array}$ & $27,27 \%$ & $\begin{array}{l}\text { Manifestações } \\
\text { de pele }\end{array}$ & $4,54 \%$ \\
\hline $\begin{array}{l}\text { Manifestações } \\
\text { gastrointestinais } \\
\text { e de pele }\end{array}$ & $13,63 \%$ & \multirow{2}{*}{$\begin{array}{l}\text { Manifestações } \\
\text { respiratórias }\end{array}$} & \multirow{2}{*}{$0,0 \%$} \\
\hline $\begin{array}{l}\text { Manifestações } \\
\text { respiratórias e } \\
\text { de pele }\end{array}$ & $4,54 \%$ & & \\
\hline
\end{tabular}


A avaliação do estado nutricional, através da análise do escore $Z$ do IMC pela idade, evidenciou eutrofia em $81,8 \%$ das crianças, risco de sobrepeso em 4,5\%, sobrepeso em $9,1 \%$ e obesidade em $4,5 \%$. Nenhum dos pacientes apresentou diagnóstico nutricional de magreza.

A ferritina sérica da maioria das crianças $(95,5 \%)$ apresentou-se normal, mesmo que $68,18 \%$ de todas elas não estavam em uso de sulfato ferroso. Todos os pacientes (100\%) com alergia em remissão e alergia resolvida mostraram nível de ferritina sérica normal, porém, $14,3 \%$ das crianças em alergia ativa estavam com esse valor abaixo do recomendado para idade.

Os pacientes que apresentaram sintomas gastrointestinais $(90,9 \%)$ tinham idade média de $3,0 \pm 1,72$ anos, $65,0 \%$ eram do sexo masculino, a maioria estava eutrófico $(85,0 \%)$ e todos $(100 \%)$ apresentaram exame de ferritina sérica normal. Já as crianças que tinham manifestações de pele $(54,4 \%)$, possuíam idade média de $2,89 \pm 1,8$ anos, $66,7 \%$ eram do sexo masculino, $75,0 \%$ apresentaram eutrofia e $91,7 \%$ apontaram valor de ferritina sérica normal. $E$ os pacientes que possuíam sintomas respiratórios $(63,6 \%)$ tinham idade média de $2,99 \pm 1,37$ anos, $64,3 \%$ eram do sexo masculino, $71,4 \%$ estavam eutróficos e todos (100\%) também apresentaram exame de ferritina sérica normal.

Referente ao tipo de leite utilizado, os percentuais de crianças em uso de fórmula infantil à base de aminoácidos (AA) foi de $31,8 \%, 13,6 \%$ de fórmula infantil à base de soja, $27,3 \%$ de fórmula infantil extensamente hidrolisada (FEH), 4,5\% de fórmula infantil à base de arroz, $9,1 \%$ de leite de soja e $13,6 \%$ a criança não tomava nenhum um tipo de leite ou estava em aleitamento materno, com exclusão de leite de vaca da dieta da mãe (Tabela 2 ).

Tabela 2. Comparação entre o leite utilizado e o diagnóstico nutricional, de acordo com o IMC das crianças com alergia a proteína do leite de vaca, atendidas no Ambulatório de Pediatria do Hospital de Clínicas da Universidade Federal do Triângulo Mineiro, Uberaba (2018). $(n=22)$

\begin{tabular}{lcccc}
\hline \multicolumn{1}{c}{$\begin{array}{c}\text { Leite } \\
\text { utilizado }\end{array}$} & \multicolumn{3}{c}{ Diagnóstico Nutricional } \\
\hline & Eutrofia & $\begin{array}{c}\text { Risco de } \\
\text { sobrepeso }\end{array}$ & Sobrepeso & Obesidade \\
AA & $33,3 \%$ & $100 \%$ & $0 \%$ & $0 \%$ \\
$\begin{array}{l}\text { FEH } \\
\text { À base de }\end{array}$ & $22,2 \%$ & $0 \%$ & $50 \%$ & $100 \%$ \\
$\begin{array}{l}\text { arroz } \\
\text { À base de } \\
\text { soja }\end{array}$ & $5,6 \%$ & $0 \%$ & $0 \%$ & $0 \%$ \\
$\begin{array}{l}\text { Leite de } \\
\text { soja }\end{array}$ & $16,7 \%$ & $0 \%$ & $0 \%$ & $0 \%$ \\
Outros & $16,6 \%$ & $0 \%$ & $50 \%$ & $0 \%$ \\
\hline
\end{tabular}

Fonte: autores (2018)
Em relação ao histórico alimentar, $63,6 \%$ das mães dos pacientes ofereceram o aleitamento materno exclusivo (AME), com duração média de 4,79 $\pm 1,88$ meses (Tabela 3 ). Já a idade média de introdução de alimentação complementar foi de $6,95 \pm 4,32$ meses.

Tabela 3. Comparação entre a duração do AME e os sintomas apresentados pelas crianças com alergia à proteína do leite de vaca, atendidas no Ambulatório de Pediatria do Hospital de Clínicas da Universidade Federal do Triângulo Mineiro, Uberaba (2018). $(n=22)$

\begin{tabular}{lcc}
\hline $\begin{array}{l}\text { Duração do } \\
\text { AME }\end{array}$ & $\begin{array}{c}\text { Número de } \\
\text { crianças em } \\
\text { AME }\end{array}$ & $\begin{array}{c}\text { Tipo de sintomas } \\
\text { apresentados }\end{array}$ \\
\hline $4,42 \pm 1,73$ & 12 & Gastrointestinais \\
$4,44 \pm 2,00$ & 9 & Respiratórios \\
$5,44 \pm 1,51$ & 9 & De pele \\
\hline
\end{tabular}

Fonte: autores (2018)

De acordo com a avaliação do comportamento alimentar de todos os participantes da pesquisa, através do questionário de frequência alimentar, constatou-se que o consumo de alimentos fonte de ferro heme era adequado, visto que a frequência de ingestão de bife $(63,6 \%)$, carne cozida $(90,9 \%)$ e frango $(86,4 \%)$ era de 2 a 4 vezes na semana, com exceção do bife de fígado, em que $54,5 \%$ das crianças nunca comeram e somente $13,6 \%$ ingeriam uma vez por semana. A respeito do consumo de legumes e verduras, também se observou adequação, pois a maioria dos pacientes comia de 2 a 4 vezes por semana esses alimentos $(60,22 \%)$, com exceção da alface, e diferentes frutas pelo menos uma vez por dia ou de 2 a 4 vezes por semana (56,06\%). Dentre todas as frutas citadas no questionário, apenas 0 mamão e a goiaba não mostraram boa aceitação pelas crianças.

Quanto ao consumo dos alimentos industrializados incluídos no questionário, tais como, biscoitos com e sem recheio, cereal matinal, macarrão instantâneo, iogurte de frutas, Danoninho ${ }^{\circledR}$, leite fermentado, achocolatado, chocolate, salgadinhos, refrigerante e suco artificial, considerou-se concordante com o que se recomenda para essa idade, visto que a maior parte das crianças $(65,15 \%)$ nunca consumiram esses tipos de alimentos. A Tabela 4 mostra a frequência de consumo de alimentos que contêm ou podem conter LV, pelos três grupos avaliados. 
Tabela 4. Frequência de consumo de alimentos que contêm ou podem conter LV em sua composição pelos pacientes que apresentavam alergia ativa, em remissão ou resolvida, Uberaba (2018). $(n=22)$

\begin{tabular}{|c|c|c|c|c|c|c|}
\hline & $\begin{array}{c}\text { Alergia } \\
\text { ativa }(n=7)\end{array}$ & $\begin{array}{l}\text { Frequência } \\
\text { de consumo }\end{array}$ & $\begin{array}{c}\text { Alergia em } \\
\text { remissão } \\
(n=3)\end{array}$ & $\begin{array}{l}\text { Frequência } \\
\text { de consumo }\end{array}$ & $\begin{array}{c}\text { Alergia } \\
\text { resolvida }\end{array}$ & $\begin{array}{l}\text { Frequência } \\
\text { de consumo } \\
(n=12)\end{array}$ \\
\hline Biscoitos com recheio & $14,3 \%$ & $\begin{array}{c}1 \text { a } 3 \text { vezes/ } \\
\text { mês }\end{array}$ & $33,3 \%$ & $\begin{array}{l}2 \text { a } 4 \text { vezes/ } \\
\text { semana }\end{array}$ & $8,3 \%$ & $1 \mathrm{vez} / \mathrm{semana}$ \\
\hline Biscoitos sem recheio & $28,6 \%$ & $\begin{array}{l}1 \text { ou mais } \\
\text { vezes/dia }\end{array}$ & $66,6 \%$ & $\begin{array}{c}\text { Pelo menos } \\
2 \text { a } 4 \text { vezes/ } \\
\text { semana }\end{array}$ & $83,3 \%$ & $\begin{array}{c}\text { Pelo menos } 2 \text { a } 4 \\
\text { vezes/semana }\end{array}$ \\
\hline Cereal matinal & $14,3 \%$ & < 1 vez/mês & $33,3 \%$ & $\begin{array}{c}1 \text { a } 3 \text { vezes/ } \\
\text { mês }\end{array}$ & $0 \%$ & - \\
\hline Iogurte/Danoninho $®$ & $28,6 \%$ & $\begin{array}{c}1 \text { a } 3 \text { vezes/ } \\
\text { mês }\end{array}$ & $0 \%$ & - & $50,0 \%$ & $\begin{array}{c}1 \text { a } 4 \text { vezes/ } \\
\text { semana }\end{array}$ \\
\hline Leite fermentado & $14,3 \%$ & $\begin{array}{c}1 \text { a } 3 \text { vezes/ } \\
\text { mês }\end{array}$ & $0 \%$ & - & $16,7 \%$ & $\begin{array}{c}2 \text { a } 4 \text { vezes/ } \\
\text { semana }\end{array}$ \\
\hline Achocolatado & $0 \%$ & - & $0 \%$ & - & $16,7 \%$ & 1 a 3 vezes/mês \\
\hline Chocolate & $28,6 \%$ & $\begin{array}{c}\text { Pelo menos } \\
1 \text { a } 3 \text { vezes/ } \\
\text { mês }\end{array}$ & $33,3 \%$ & $\begin{array}{c}1 \text { a } 3 \text { vezes/ } \\
\text { mês }\end{array}$ & $33,4 \%$ & $\begin{array}{c}1 \text { a } 4 \text { vezes/ } \\
\text { semana }\end{array}$ \\
\hline Margarina/Manteiga & $57,2 \%$ & $\begin{array}{l}\text { Pelo menos } \\
2 \text { a } 4 \text { vezes/ } \\
\text { semana }\end{array}$ & $100 \%$ & $\begin{array}{c}\text { Pelo menos } \\
1 \text { a } 3 \text { vezes/ } \\
\text { mês }\end{array}$ & $41,6 \%$ & $\begin{array}{c}\text { Pelo menos } 2 \text { a } 4 \\
\text { vezes/semana }\end{array}$ \\
\hline Queijo & $14,3 \%$ & $\begin{array}{c}1 \text { a } 3 \text { vezes/ } \\
\text { mês }\end{array}$ & $33,3 \%$ & $1 \mathrm{vez} / \mathrm{semana}$ & $16,6 \%$ & $\begin{array}{l}2 \text { a } 4 \text { vezes/ } \\
\text { semana }\end{array}$ \\
\hline Requeijão & $0 \%$ & - & $33,3 \%$ & $1 \mathrm{vez} / \mathrm{semana}$ & $16,6 \%$ & $\begin{array}{l}2 \text { a } 4 \text { vezes/ } \\
\text { semana }\end{array}$ \\
\hline Bolos & $42,85 \%$ & $\begin{array}{c}\text { Pelo menos } \\
1 \text { a } 3 \text { vezes/ } \\
\text { mês }\end{array}$ & $100 \%$ & $\begin{array}{c}\text { Pelo menos } \\
1 \text { a } 3 \text { vezes/ } \\
\text { mês }\end{array}$ & $58,3 \%$ & $\begin{array}{l}1 \text { a } 4 \text { vezes/ } \\
\text { semana }\end{array}$ \\
\hline
\end{tabular}

Source: authors (2018)

\section{DISCUSSÃO}

É importante destacar algumas limitações do estudo, como o número reduzido da amostra e a ausência dos pacientes nas consultas. $O$ ambulatório atende poucos pacientes com APLV e estes faltam muito às consultas, o que impossibilitou a coleta de dados.

A pesquisa revelou um maior percentual de crianças do sexo masculino, da mesma forma que a pesquisa publicada pela Revista Paulista de Pediatria, demonstrando um percentual de 58,4\% de meninos com APLV. ${ }^{26}$

O estudo identificou que a maior parte dos participantes $(63,6 \%)$ não foi amamentada exclusivamente até os seis meses; e começaram a receber a alimentação complementar a partir dessa idade. Resultados semelhantes foram identificados em estudo realizado em um ambulatório infantil de Salvador, onde somente $7,5 \%$ das crianças avaliadas estiveram em AME até os seis meses. ${ }^{27}$ No presente estudo foi possível observar uma média de 4,8 meses em relação ao tempo de aleitamento materno exclusivo na população estudada.

Esses dados contrariam as recomendações do Guia Alimentar para Crianças Brasileiras Menores de Dois Anos (2019) ${ }^{28}$ sobre o aleitamento materno exclusivo até os 6 meses, entretanto, a média da introdução alimentar encontra-se dentro da faixa de idade recomendada para início da alimentação complementar. Ainda sobre o aleitamento materno, de acordo com o Consenso Brasileiro sobre Alergia Alimentar $(2018)^{22}$, ele é considerado um fator protetor no desenvolvimento de alergias alimentares e deve ser preconizado.

Com relação à avaliação do estado nutricional, observou-se que a maior parte da amostra apresentou adequação aos parâmetros avaliados, o que corrobora com a hipótese de que a dieta de substituição está sendo realizada de forma correta ou de que a fórmula tem atendido as necessidades nutricionais das crianças. $O$ estudo de Brito et al. ${ }^{29}$, realizado com crianças de 0 a 5 anos de idade com 
diagnóstico de APLV, indicou resultados parecidos: quase $90 \%$ dos participantes apresentaram IMC por idade adequado. Já em outro estudo, casocontrole, na comparação dos grupos, as crianças com APLV apresentaram menor peso corporal e menor comprimento por estatura. ${ }^{30}$ Outra pesquisa avaliou somente crianças com APLV e verificou, pelo IMC para idade, que $12,9 \%$ das crianças apresentavam magreza ou magreza acentuada, $67,8 \%$ eutrofia, $15,2 \%$ risco para sobrepeso e $4,1 \%$ sobrepeso ou obesidade. ${ }^{26}$ Contudo, neste estudo, nenhum dos pacientes apresentou diagnóstico nutricional de magreza, reforçando que o uso das fórmulas e a alimentação estavam de acordo com as necessidades de cada paciente. Os resultados também indicaram que parte dos participantes estava com risco de sobrepeso $(4,5 \%)$ e sobrepeso e obesidade $(13,6 \%)$. Não foram encontrados estudos que relacionassem APLV e sobrepeso e obesidade, nem uma possível relação destes com o uso de fórmulas infantis por pacientes com APLV. Porém, na amostra estudada, verificou-se que muito cedo as crianças começaram a receber alimentos inadequados, como doces industrializados, biscoitos recheados, macarrão instantâneo, entre outros; tais práticas podem levar ao aumento dos riscos de desenvolvimento de doenças crônicas como, por exemplo, a obesidade, apesar dos dados do presente estudo mostrarem que o consumo desse tipo de alimento é baixo.

Os pacientes com diagnóstico de APLV são orientados a ingerir substitutos que contenham alto teor proteico e que permitam um bom desenvolvimento. De acordo com o estudo de Solé et al. ${ }^{11}$, antes das fórmulas industrializadas com proteínas hidrolisadas ou à base de aminoácidos, eram utilizados leites de outros mamíferos; mostrando-se inadequado em função do aparecimento de outras manifestações clínicas adversas após sua ingestão. Os participantes deste estudo não fazem uso de leite de outros mamíferos, mas de fórmulas $A A$, $\mathrm{FEH}$, fórmula infantil à base de soja, de arroz, leite de soja e outros, ou não tomavam nenhum tipo de leite ou estavam em aleitamento materno. O estudo realizado em uma Unidade de Saúde de Belém, verificou que as fórmulas mais fornecidas são a fórmula à base de aminoácidos (47.1\%) e a fórmula à base de proteína extensamente hidrolisada (42.9\%). ${ }^{31}$ Brito et al..$^{29}$ também observaram o maior consumo desses dois tipos de fórmulas infantis para substituição do LV. Já a pesquisa de Vandenplas ${ }^{32}$, evidenciou o uso de fórmula à base de proteína extensamente hidrolisada como a primeira opção de escolha, a fórmula à base de arroz e fórmula à base de soja como opções de segunda escolha e o uso de fórmulas de aminoácidos somente para casos mais graves. As fórmulas infantis são seguras nutricionalmente, podendo variar o custo, sendo algumas mais caras, como as AA e outras mais baratas; além da questão da palatabilidade. ${ }^{33}$ Estes fatores, segundo a literatura, juntamente com as indicações dos profissionais, orientam os pais na hora de escolher e de como administrá-las.

A alimentação complementar descrita pelos participantes mostrou-se adequada, visto que a maioria dos pacientes consome quantidades adequadas de hortaliças e de alimentos fonte de ferro heme. De acordo com Alves et al. ${ }^{34}$, a alimentação complementar de crianças com APLV deve seguir os mesmos princípios que são preconizados para crianças saudáveis, não havendo necessidade de restringir alimentos com proteínas alergênicas, tais como ovo e peixe. Segundo as recomendações do Ministério da Saúde, é importante um consumo variado de alimentos dentro de um mesmo grupo. ${ }^{35}$ Entretanto, foi observada a presença de produtos alimentícios que contêm ou podem conter LV na rotina alimentar de algumas crianças que estavam na fase de alergia ativa, o que contraria a recomendação principal para o tratamento da APLV, que é a exclusão de LV e derivados da alimentação. ${ }^{22}$

Em princípio, na alergia alimentar pode ocorrer deficiência de ferro em função de perdas fecais ou de má absorção secundária à lesão do intestino delgado ou da inflamação sistêmica. Embora não seja um exame para diagnóstico de alergia, como APLV, o hemograma auxilia na deteç̧ão de complicações a ele associados, como a anemia. Nesse estudo, foram analisados os resultados do valor de ferritina sérica e o uso de suplementação de sulfato ferroso dos participantes do estudo. De modo geral, a ferritina sérica apresentou-se normal, sendo que a maioria não estava em uso de sulfato ferroso. Entretanto, $14,3 \%$ das crianças em alergia ativa apresentaram esse valor abaixo do indicado para idade. Para esses casos, ressalta-se a importância de novas investigações e novas orientações sobre alimentação e uso de sulfato ferroso, por exemplo.

Nos casos de tratamento de APLV, o nutricionista e o médico pediatra devem estar atentos às faixas de 
idade que esta patologia pode acometer e manteremse atualizados com as terapêuticas e diagnósticos, provendo as necessidades desses infantes de acordo com as recomendações diárias individuais. Além disso, devem ensinar aos pais e aos cuidadores a leitura e interpretação de rótulos dos produtos alimentícios, pois intervenções errôneas poderão causar agravos persistentes. Os dados encontrados nesse estudo indicam que os responsáveis seguem as orientações dos profissionais, uma vez que não foram observados casos de manifestações dos sintomas de APLV por causa de uma dieta de substituição inadequada. ${ }^{36}$

Somente profissionais de saúde podem indicar as fórmulas infantis para crianças com necessidades alimentares especiais, como é o caso de crianças com APLV, assim como suas formas de preparo e as quantidades a serem oferecidas individualmente. Eles também poderão orientar se é necessária a suplementação de micronutrientes. ${ }^{28}$

\section{CONCLUSÃO}

Conclui-se que as crianças com APLV apresentam como manifestação da doença, predominantemente, sintomas gastrointestinais. $O$ estado nutricional encontra-se adequado e não apresentam anemia por deficiência de ferro. Quanto ao padrão alimentar, a substituição do LV é realizada principalmente por fórmulas infantis $A A$ e $\mathrm{FEH}$; há consumo variado e adequado de hortaliças e carne vermelha, baixo consumo de alimentos industrializados, e concordante período de início da alimentação complementar, porém, desmame precoce do leite materno.

\section{REFERÊNCIAS}

1. Motala C, Fiocchi A. Cow's milk allergy in children. World Allergy Organ J [serial on the Internet]. 2012 [cited 2012 Jul]. Available from: http://www.worldallergy. org/professional/allergic_diseases_center/cows_milk_ allergy_in_children/.

2. Vandenplas $Y$, Castrellon PG, Rivas R, Gutiérrez C, Garcia LD, Jimenez JE, et al. Safety of soya-based infant formulas in children. Br J Nutr. 2014 Apr 28; 111(8):1340-60.

3. Sicherer, SH. Epidemiology of food allergy. J Allergy Clin Immunol. 2011 Mar; 127 (3):594-602.
4. Maia ALGL. Aleitamento materno em crianças com e sem alergia à proteína do leite de vaca. Orientadora: Silvia Alves da Silva. 2019. Monografia (Trabalho de Conclusão do Curso de Nutrição). Universidade Federal de Pernambuco, Vitória de Santo Antão, 2019. Disponível em: https://repositorio.ufpe.br/ handle/123456789/33840.

5. Brasil. Ministério da Saúde. Comissão Nacional de Incorporação de Tecnologias no SUS. Protocolo Clínico e Diretrizes Terapêuticas Alergia à Proteína do Leite de Vaca (APLV) [Internet]. 2017 [citado 2020 jan. 13]. Disponível em: http://conitec.gov.br/images/ Consultas/ Relatorios/2017/Relatorio_PCDT_APLV_CP68_2017.pdf.

6. Alves JQ, Mendes JF, Jaborandy ML. Perfil nutricional e consumo dietético de crianças alérgicas à proteína do leite de vaca acompanhadas em um hospital infantil de Brasília/DF, Brasil. Com. Ciências Saúde. 2017; 28(3/4):402-412

7. Sardecka I, Łoś-Rycharska E, Ludwig H, Gawryjołek J, Krogulska A. Early risk factors for cow's milk allergy in children in the first year of life. Allergy and Asthma Proceedings, 2018; 39(6): e44-e54.

8. Silva AML, Monteiro GRSS, Tavares ANA, Pedrosa ZVRS. The early food introduction and the risk of allergies: A review of the literature. Enfermería Global, 2019; 59: 499-511.

9. Kansu A, Yüce A, Dalgıç B, Şekerel BE, Çullu-Çokuğraş $F$, Çokuğraş $H$. Consensus statement on diagnosis, treatment and follow-up of cow's milk protein allergy among infants and children in Turkey. Turk J Pediatr. 2016; 58(1):1-11.

10. Kwan A, Asper M, Lavi S, Lavine E, Hummel D, Upton JE. Prospective evaluation of testing with baked milk to predict safe ingestion of baked milk in unheated milkallergic children. Allergy Asthma Clin Immunol. 2016 Oct $24 ; 12(54): 1-8$.

11. Solé D, Amancio OMS, Jacob CMA, Cocco RR, Sarni ROS, Suano F. Guia prático de diagnóstico e tratamento da alergia às proteínas do leite de vaca mediada pela imunoglobulina E. Rev. bras. alerg. imunopatol. 2012;35(6):203-33.

12. Solé $D$, Silva LR, Cocco RR, Ferreira CT, Sarni RO, Oliveira LC et al. Consenso Brasileiro sobre Alergia Alimentar: 2018 - Parte 2 -Diagnóstico, tratamento e prevenção. Arquivos de Asma, Alergia e Imunologia, 2018; 2(1): 39-82.

13. Luyt D, Ball H, Makwana N, Green MR, Bravin K, Nasser $\mathrm{S} M$ et al. Standard of Care Committee (SOCC) of the British Society for Allergy and Clinical Immunology (BSACI). BSACI guideline for the diagnosis and management of cow's milk allergy. Clin. Exp. Allergy 2014, 44, 642-672.

14. Brasil. Ministério da Saúde. Cadernos de atenção básica: Saúde da criança - Aleitamento materno e alimentação complementar [livro online]. Brasília, DF: Ministério da Saúde, 2015. Disponível em: http://bvsms.saude. gov.br/bvs/publicacoes/saude_crianca_aleitamento_ materno_cab23.pdf 
15. Morais MB, Speridião, PGL, Sillos, MD. Alergia à proteína do leite de vaca. RBM rev. bras. med. [serial on the Internet]. 2013; 49(8) [cited 2013 Aug]. Available from: http://www.moreirajr.com.br/revistas. asp?fase $=$ r003\&id_materia $=5465$

16. Sarubbi V, Mylaert CJ, Bastos IT, Gallo PR, Leone C. Representações de pediatras acerca das alternativas de alimentos lácteos diante do desmame inevitável. Rev Paul Pediatr, 2017; 35(1):46-53.

17. Sociedade Brasileira de Nutrição Parenteral e Enteral, Sociedade Brasileira de Clínica Médica, Associação Brasileira de Nutrologia. Projeto Diretrizes: Terapia Nutricional no Paciente com Alergia ao Leite de Vaca. Associação Médica Brasileira e Conselho Federal de Medicina. [serial on the Internet] 2011 [cited 2011 Jul 11]. Available from: https://diretrizes.amb.org. br/_BibliotecaAntiga/terapia_nutricional_no_paciente_ com_alergia_ao_leite_de_vaca.pdf

18. Pensabene L, Salvatore S, D'Auria E, Parisi S, Concolino $D$, Borrelli $O$ et al. Cow's Milk Protein Allergy in Infancy: A Risk Factor for Functional Gastrointestinal Disorders in Children? Nutrients, 2018; 10(11), 1716.

19. Sánchez-Valverde F, Etayo V, Gil F, Aznal E, Martínez $D$, Amézqueta $A$ et al. Factors Associated with the Development of Immune Tolerance in Children with Cow's Milk Allergy. Int Arch Allergy Immunol, 2019; 179:290-296.

20. Brito CT, Silva JVE, Garcia LRS. Perfil nutricional de crianças atendidas pelo programa de controle de alergia à proteína do leite de vaca no município de Natal/RN. Revista Humano Ser - UNIFACEX, 2017, 3(1):1-18.

21. Heine RG, AlRefaee F, Bachina P, Leon JC, Geng L, Gong $S$ et al. Lactose intolerance and gastrointestinal cow's milk allergy in infants and children-common misconceptions revisited. World Allergy Organization Journal. 2017; 10(1):41.

22. Solé $D$, Silva LR, Cocco RR, Ferreira CT, Sarni RO, Oliveira LC, et al. Consenso Brasileiro sobre Alergia Alimentar: 2018 - Parte 1 - Etiopatogenia, clínica e diagnóstico. Documento conjunto elaborado pela Sociedade Brasileira de Pediatria e Associação Brasileira de Alergia e Imunologia.Arq Asma Alerg Imunol. 2018;2(1):7-38.

23. World Health Organization Multicentre Growth Reference Study Group. WHO Child Growth Standards: Length/height-for-age, weight-for-age, weight-forlength, weight-for-height and body mass index-for-age: Methods and development. World Health Organization. [serial on the Internet] 2006 [cited 2006]. Available from: https://www.who.int/childgrowth/standards/ Technical_report.pdf?ua=1

24. Colucci ACA, Philippi ST, Slater B. Development of a food frequency questionnaire for children aged 2 to 5 years. Rev. bras. epidemiol. 2004 dec;7(4).

25. Sociedade Brasileira de Pediatria. Manual de orientação: alimentação do lactente, alimentação do pré-escolar, alimentação do escolar, alimentação do adolescente, alimentação na escola. Departamento de Nutrologia. [serial on the Internet] 2006 [cited 2006]. Available from: http://www.sbp.com.br/fileadmin/user_upload/ img/manuais/manual_alim_dc_nutrologia.pdf

26. Aguiar ALO, Maranhão CM, Spinelli LC, Figueiredo RM, Maia JMC, Gomes RC, et al. Avaliação clínica e evolutiva de crianças em programa de atendimento ao uso de fórmulas para alergia à proteína do leite de vaca. Rev Paul Pediatr. 2013; 31:152-158.

27. Oliveira LS, Santos DSM, Paixão CGO. Hábitos alimentares de crianças menores de dois anos com alergia à proteína do leite de vaca. Rev. bras. de saúde funcional. 2019 ago; 8(1).

28. Brasil. Ministério da Saúde. Secretaria de Atenção Primária à Saúde. Departamento de Promoção à Saúde. Guia alimentar para crianças brasileiras menores de 2 anos. Brasília, DF: Ministério da Saúde, 2019. Disponível em: http://189.28.128.100/dab/docs/portaldab/ publicacoes/guia_da_crianca_2019.pdf.

29. Brito HCA, Brandão HFC, Lins TIS, Neves CMAF, Macêdo $J N$, Silva DRLS. Estado nutricional e hábitos alimentares de crianças diagnosticadas com alergia a proteina do leite de vaca em dieta de exclusão. Br J D. 2021 jan; 7(1):10029-42.

30. Korz V, Kremr MM, Vargas DM, Nunes CRO. Cow's milk protein allergy, quality of life and parental style. $\mathrm{J}$ Hum Growth Dev. 2021; 31(1):28-36.

31. MENDONÇA KRC, Nascimento LP, Mansour IB, Silva ALFA, Nobre ALN, Nassimento LP et al. Custos diretos dos responsáveis pelos pacientes portadores de alergia alimentar à proteína do leite de vaca em uma unidade básica de saúde em Belém Braz. J. Hea. Rev. 2020 set/ out; 3(5):12684-99.

32. VANDENPLAS Y. Prevention and Management of Cow's Milk Allergy in Non-Exclusively Breastfed Infants. Nutrients, 2017; 9:731.

33. Yonamine GH, Castro APBM, Pastorino AC, Jacob CMA. Uso de fórmulas à base de soja na alergia à proteína do leite de vaca. Rev. Bras. Alerg. Imunopatol., 2011; 34: 187-92.

34. Alves JQN, Mendes JFR, Jaborandy ML. Perfil nutricional e consumo dietético de crianças alérgicas à proteína do leite de vaca acompanhadas em um hospital infantil de Brasília/DF, Brasil. Comunicação em Ciências da Saúde, 2018; 28(03 04):402-412.

35. Brasil, Ministério da Saúde. Estudo Nacional de Alimentação e Nutrição Infantil (ENANI). Brasília, DF: Ministério da Saúde, 2020. Disponível em: https:// www.saude.gov.br/noticias/agencia-saude/47311pesquisa-inedita-revela-que-indices-de-amamentacaocresceram-no-brasil

36. Santos BO, Lima LFD. Galactosemia, intolerância à lactose e alergia à proteína do leite: compreensão dos mecanismos fisiopatológicos na primeira infância e suas respectivas prescrições nutricionais. Temas em Educ. e Saúde, 2020; 16(2): 500-512. 
Autor Correspondente:

Maísa Tirintan Jordani

maisajordani@hotmail.com

Editor:

Prof. Dr. Marcelo Riberto

Recebido: 20/10/2020

Aprovado: 25/06/2021 